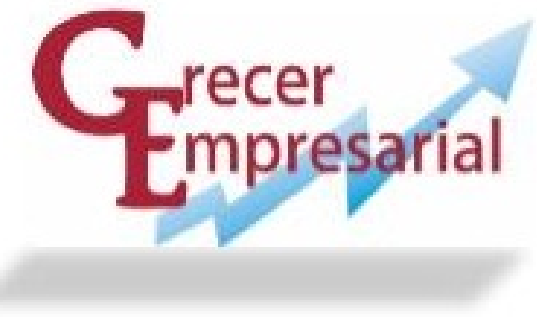

\title{
LA COMPETITIVIDAD DEL TALENTO HUMANO EN EL AMBITO EMPRESARIAL
}

\author{
Pérez Vásquez Manuel Antonio ${ }^{1}$
}

Artículo Enviado: 30/05/2017; Aceptado: 10/01/2019; Publicado: 11/02/2019

\begin{abstract}
Resumen
La gestión por competencias del talento humano, debe ser comprendida desde la actuación del empleado o trabajador dentro de una entidad privada o pública, donde es relevante, la mejora del desempeño y los aportes del personal en el marco de una actividad ética y socialmente responsable. La gestión por competencias del talento humano ha tomado gran relevancia en la actualidad, por ello, es valioso que exista un conocimiento adecuado sobre esta área funcional en cada organización. Este trabajo hace énfasis en los elementos que influyen sobre las competencias del talento humano en las organizaciones desde su desarrollo teórico, donde se aborda los conceptos y teorías del talento humano y la gestión por competencias; de igual modo, en la discusión se presentan los resultados del estudio empírico realizado sobre el desarrollo del talento humano en varias empresas en la ciudad de Bogotá, con el objetivo de contrastar lo descrito conceptualmente.
\end{abstract}

Palabras claves: Empresarial, competitividad, talento humano, gestión

\section{THE COMPETITIVENESS OF HUMAN TALENT IN THE BUSINESS AREA}

\begin{abstract}
Management by skills of human talent should be understood from the employee performance inside a private or public entity organization where it is relevant, the improvement of the performance and contributions of the staff, where it is relevant the improvement of the performance and contributions of the staff within of an ethical and socially responsible activity. Management by skills of human talent has taken great relevance today, thus, it is valuable that it exists the suitable knowledge about this functional area in each organization. This research emphasizes the elements that influence the skills of human talent in organizations since their theoretical development, where the concepts and theories of human talent and skills management are addresse, in the same way, the discussion presents the results of the empirical study accomplished about the development of human talent in several companies in Bogota city, in order to contrast the conceptually described.
\end{abstract}

\footnotetext{
${ }^{1}$ Universidad del Sinú. Email: manuelperezv@unisinu.edu.co
}

PEREZ VASQUEZ, M.A. (2019).

Este es un artículo open Access bajo Licencia Creative Commons Atribución-No Comercial-Sin Derivadas 4.0 Internacional. 
Keywords: business, competitiveness, human talent, management.

\section{LA COMPÉTITIVITÉ DES TALENTS HUMAINS DANS L'ENVIRONNEMENT DES ENTREPRISES}

\section{Résumé}

La gestion par compétences de talent humain doit être comprise à partir de la performance du salarié ou de l'employé au sein d'une entité privée ou publique, où est-ce pertinent, l'amélioration des performances et de la contribution du personnel à l'organisation, dans le cadre d'une activité éthique et socialement responsable. La gestion par compétences du talent humain a pris une grande pertinence dans l'actualité, il est donc précieux qu'il y ait une connaissance adéquate de ce domaine fonctionnel dans chaque organisation. Dans cet article, l'accent est mis sur les éléments qui influencent les compétences du talent humain dans les organisations, leur développement théorique, présente l'état de la l'art où sont abordés les concepts et les théories du talent humain et de la gestion par compétences. De la même manière, la discussion montre les résultats d'une étude empirique réalisée sur le développement du talent humain dans plusieurs entreprises de la ville de Bogotá, dans le but de contraster le concept décrit et ainsi de générer finalement quelques conclusions de l'étude.

Mots-clés: entrepreneurial, compétitivité, talent humain, management.

\section{A COMPETIVIDADE DO TALENTO HUMANO NO AMBIENTE EMPRESARIAL}

\section{Resumo}

A gestão pela competência do talento humano, deve ser compreendida desde a atuação do empregado ou trabalhador dentro de uma entidade particular ou pública, onde é relevante, a melhora do desempenho e os aportes do trabalhador à organização, no marco de uma atividade ética e socialmente responsável. A gestão pela competência do talento humano, tinha tomado mais relevância na atualidade, por tanto, é valioso que exista um conhecimento adequado sobre esta área funcional em cada organização. Neste artigo, se faze ênfases em aqueles elementos que influenciam sobre as competências do talento humano nas organizações, o desenvolvimento teórico, apresenta o estado da arte onde se aborda os conceitos e as teorias do talento humano e a gestão pelas competências. Do mesmo jeito, na discução se monstra os resultados de um estudo empírico realizado sobre o desenvolvimento do talento humano em várias empresas na cidade de Bogotá, com o objetivo de contrastar o descrito conceitualmente e assim, finalmente gerar umas conclusões do estudo.

Palavras chaves: empresarial, competitividade, talento humano, gestão

PEREZ VASQUEZ, M.A. (2019).

Este es un artículo open Access bajo Licencia Creative Commons Atribución-No Comercial-Sin Derivadas 4.0 Internacional. 


\section{Introducción}

Las empresas en Colombia se caracterizan en su mayoría, por su informalidad y poca asertividad, al momento de describir las reales funciones y la adecuada naturaleza de los cargos que existen en cada una de ellas. Dicha situación, hace que sea menos competitivo el recurso humano que poseen. Las organizaciones, al no estar bien definidas estructuralmente (es decir, en cuanto a las funciones y los procedimientos operativos), son débiles y se encuentran en desventaja frente a empresas que son su competencia.

Es muy común observar, que las empresas no hacen un uso adecuado de manuales, procedimientos y normatividades internas que permitan una mejor administración del recurso humano, se dedican a establecer lineamientos cortoplacistas de dirección inmediata, sin llegar a la etapa previsiva y mucho menos, a la planificación de las actividades futuras.

Otra falencia importante que se debe señalar en las empresas colombianas, es la informalidad en la toma de decisiones y en los procesos de vinculación laboral, donde se desconoce todo el proceso de selección, contratación de personal y adaptación laboral. También, es característico en muchas de estas organizaciones que su direccionamiento sea ejecutado por una sola persona, quien, comúnmente es el gerente (ayudado por varios colaboradores que son netamente familiares), porque se genera desconfianza en delegar funciones de tipo administrativo a otras personas, por parte de gerentes-propietarios.

El proceso de gestión del talento humano, se refiere a planear y organizar la estructura del personal y cargos que componen la empresa, así como dirigir y controlar sus actividades. Se ha comprobado, que la eficiencia de la empresa es mayor que la suma de las eficiencias de los trabajadores, y que ella debe alcanzarse mediante la racionalidad ${ }^{2}$.

Por otra parte, la nueva tendencia en el plano de la competitividad del recurso humano, es considerar al personal que labora en las empresas como un cliente más, en el sentido, de comprender que las personas son la prioridad en una organización. Los colaboradores deben ser tratados como clientes, lo que significa comprender sus necesidades, y así lograr el desempeño adecuado en sus labores. Se considera, que, si el trabajador está estable emocionalmente, realizará sus actividades de manera adecuada, por lo tanto, atenderá a los clientes de la mejor manera posible, logrando la fidelización del mismo.

Con lo anterior, este trabajo se basó en resolver la pregunta: ¿En qué medida, la gestión del talento humano se convierte en una herramienta estratégica para la competitividad de las empresas?

Se entiende que la importancia de la gestión del talento humano en las empresas, consiste en lograr que los colaboradores trabajen de manera eficaz en el logro de los objetivos, donde se debe considerar la necesidad de aumentar su capacidad productiva y sus aptitudes, al punto de que puedan valerse por sí mismos y entregar lo mejor en su trabajo, llegando a sentirse conformes con las actividades que realizan. En la actualidad, se necesita que el personal se desprenda del temor que produce lo desconocido e ingrese en la aventura de cambiar su interior a través de la innovación continua, comprendiendo la realidad y enfrentando el futuro de la compañía, considerando que lo único cierto en el mercado actual, es el cambio y la incertidumbre.

Por ello, se hace indispensable para las empresas implementar programas y estrategias para competir eficazmente en el mercado y así lograr sus objetivos organizacionales. La gestión del talento humano, es

${ }^{2}$ Es decir, a través de la adecuación de los medios humanos, materiales y técnicos a los fines que se desean alcanzar.

PEREZ VASQUEZ, M.A. (2019).

Este es un artículo open Access bajo Licencia Creative Commons Atribución-No Comercial-Sin Derivadas 4.0 Internacional. 
parte integral del proceso organizacional, incluye la detección de las necesidades de personal y el desarrollo de un programa que satisfaga las mismas.

En una empresa es importante concebir que la eficiencia de su fuerza laboral tiene su comienzo y fundamentación en el reclutamiento, selección y contratación de buenos colaboradores. Sin embargo, lo central, es la apropiada gestión del talento humano para desplegar las potencialidades del personal e incrementar considerablemente el rendimiento de la empresa para llegar a ser más competitivos en el mercado global.

Por último, el objetivo central de este trabajo, es describir la importancia de la gestión del talento humano en la organización y su incidencia en la competitividad empresarial, para lo cual, es de observancia teorizar sobre postulados de la gestión del talento humano y la gestión por competencias desde la perspectiva de la estrategia empresarial, para valorar el papel del talento humano a favor de la eficiencia y la eficacia dentro del contexto empresarial.

\section{Fundamentación Teórica}

Yusely (2018), expresa que, actualmente las organizaciones tienen como propósito central lograr que su recurso humano cumpla con las competencias específicas para ocupar los puestos de trabajo, sin embargo, es común observar que muchas empresas no conocen sus competencias y por lo tanto, no aplican nuevas estrategias direccionadas hacia el cambio en los métodos y técnicas para mejorar el desempeño, la productividad y el desarrollo de las personas. Para la autora, la gestión por competencias, implica que se establezcan índices de desempeño para cada uno de los puestos de trabajo para identificar el desarrollo en la carrera laboral de los colaboradores. La evaluación de desempeño es un elemento que sirve para la medición de la labor que desempeña un trabajador o empleado, además, sirve para tener un conocimiento de los niveles de competencias de los trabajadores en el puesto de trabajo. Finalmente, el rendimiento laboral sirve de insumo para la toma de decisiones a fin de establecer planes de mejoras en la administración del recurso humano y programas de crecimiento laboral.

Arevalo (2018), en su estudio expone que la gestión por competencias es un factor que posibilita la evaluación de las competencias personales específicas para cada puesto de trabajo en una organización. Esto, es beneficioso para el crecimiento personal de los colaboradores, debido a que ellos no solo son ocupantes de un puesto de trabajo o encargados de una labor determinada, sino que son aportantes al desarrollo de la organización. Para el autor, dicha consideración conlleva a que los dirigentes de las empresas, inviertan en las personas considerandolo altamente rentable y en concomitancia con el desarrollo personal y profesional dentro de la organización.

Según el autor, las competencias definidas por la organización pueden ser aquellas competencias cardinales, referidas a todos los colaboradores, las competencias específicas gerenciales, es decir, para aquellos que dirigen la organización y tienen otras personas a su cargo, y, por último, las competencias específicas, que son las establecidas en cada unidad funcional de la empresa.

Según Rivero, y Dabos, (2017), la diferenciación es un factor clave dentro del contexto de la dirección del recurso humano, y tal consideración, se fundamenta en el hecho de que algunos empleados aportan mayor valor a la organización. Para los autores, la diferenciación del talento humano, no solo debe estar en línea con su valor estratégico y su especificidad, sino también, con el desempeño del trabajador en su puesto de trabajo. Las distintas habilidades de los individuos y el nivel de desempeño del mismo en su puesto de trabajo son dos etapas fundamentales dentro del análisis del proceso organizacional. En ese orden de ideas, Rivero y Dabos (2017), en su estudio, determinan que se debe examinar la percepción y respuesta individual de los colaboradores, en cuanto al creciente uso de la

PEREZ VASQUEZ, M.A. (2019).

Este es un artículo open Access bajo Licencia Creative Commons Atribución-No Comercial-Sin Derivadas 4.0 Internacional. 
diferenciación de la fuerza laboral, ya que ello contribuye a la efectividad de los sistemas y prácticas de gestión del talento humano.

Según Bayona y Otros (2016), el reto actual de la dirección y gestión de los recursos humanos radica en crear herramientas útiles, mediante las cuales el personal llegue a comprometerse con los objetivos organizacionales e integrarse en el proyecto de empresa, para obtener ventajas sostenibles y duraderas en el tiempo. El compromiso organizacional, puede ser uno de los mecanismos que tiene la dirección de recursos humanos para analizar la lealtad y vinculación de los empleados con su organización. Así pues, si se consigue que los colaboradores estén identificados e implicados con la organización en la que trabajan ${ }^{3}$, mayores serán las probabilidades de que permanezcan en la misma.

Por su parte, Sum (2015), menciona que en las organizaciones la motivación es un elemento fundamental, a fin de que los colaboradores de las empresas tengan un buen desempeño laboral para lograr los objetivos propuestos y aumentar la productividad de la organización. La motivación se puede aplicar de diferentes maneras en una organización y ayuda a describir la conducta de los colaboradores en el trabajo. Asimismo, para el autor, el desempeño de los colaboradores se ve reflejado en las actividades que realizan en su área de trabajo, todo depende de la forma y la manera en que los colaboradores de una organización sean motivados.

Para Escalona (2015), en la actualidad, el mundo laboral exige muchas capacidades, habilidades y talentos, ya no es suficiente haber concluido una carrera profesional sin contar con el título correspondiente. La competencia cada vez es más reñida y la situación socioeconómica de muchos países no permite ofertar la cantidad suficiente de empleos.
Por esta razón se hace más imprescindible que los egresados de cualquier carrera cuenten con la mejor preparación y experiencia para poder destacar en ámbito laboral. Escalona plantea que el área de recursos humanos debe evolucionar, de ser un área de servicio, a ser un socio estratégico de negocio, por ende, dicha área tiene el propósito de ayudar a las personas y a la organización a lograr sus objetivos. El desarrollo estratégico del talento humano debe consolidarse como una ventaja competitiva en la organización.

Mares y Mendoza (2015), exponen que una correcta selección de personal, el clima laboral y la capacitación, son factores que influyen en la permanencia de un colaborador en una organización; a pesar de los incrementos de sueldo o las prestaciones; los empleados valoran un buen ambiente de trabajo y que las organizaciones inviertan en su capacitación y desarrollo personal. Los autores concluyen que la capacitación y el clima laboral son factores que pueden originar rotación de personal, por lo tanto, es esencial la capacitación laboral constante, y trabajar en función de un clima laboral adecuado y motivador en la organización.

Pardo y Díaz. (2014), plantean que en el desarrollo de la gestión del talento humanose debe hacer un análisis, una previsión y una potencialización del comportamiento de los colaboradores, así como la observancia de las dinámicas internas laborales y del conocimiento. Según los autores, las personas que lideran los procesos de talento humano, identifican que los cambios generan un menor impacto cuando se realizan planes de capacitación y desarrollo de competencias. De igual manera, consideran que los cambios son propicios para las organizaciones, ya que ayudan a reducir la tensión y a mejorar el clima laboral, reduciendo la incertidumbre y aumentando la participación de los equipos de trabajo en línea con la adaptación de los nuevos elementos del cambio.

${ }^{3}$ Compromiso actitudinal.

PEREZ VASQUEZ, M.A. (2019).

Este es un artículo open Access bajo Licencia Creative Commons Atribución-No Comercial-Sin Derivadas 4.0 Internacional. 
La persona encargada de la gestión del recurso humano, debe ser un evaluador del impacto y un garante de la efectividad del proceso de adaptación laboral de los colaboradores, puesto que debe estar enterado de las singularidades o particularidades presentes previas al cambio, pues tiene conocimiento de las competencias de sus colaboradores.

Para Castillo (2014), en el tratamiento del talento humano, se debe precisar acerca de la relación entre tres variables: clima organizacional, motivación intrínseca y satisfacción laboral. La psicología social y el estudio del comportamiento humano en las organizaciones, han permitido establecer dinámicas laborales específicas y explicar los orígenes del comportamiento al interior de distintos sistemas sociales; en ese sentido, las organizaciones deben tener en cuenta aquello que mejora la motivación de los trabajadores, tales como el fortalecimiento de la autonomía y el empoderamiento. De esta forma se podría implementar actividades que incluyan y fomenten estos aspectos que permitan incrementar la satisfacción laboral.

Según Caicedo y otros (2013), la empresa, en su más amplio significado, es un mundo único el cual comprende muchos factores y elementos - técnicos, materiales, financieros, infraestructura, entre otros, los cuales se deben usar adecuadamente con el fin de alcanzar las metas y conseguir los objetivos propuestos. En los elementos mencionados, se encuentran la estrategia y el talento humano, los cuales son de vital importancia para la perdurabilidad y sobrevivencia de la empresa, más aún en estos tiempos de crisis marcados por la dura competencia y el afán de diferenciación y posicionamiento, esto lleva a las empresas a reinventarse buscando nuevos mercados y modelos de negocio. Es allí, donde estos dos factores entran en juego a través de una buena gestión del talento humano y su alineación con la estrategia de la organización para lograr los cambios.

Chicaiza (2013), expone que las organizaciones al igual que los individuos poseen personalidad propia y cada organización es diferente una de la otra por ésta característica en particular. Estas se componen de un conjunto de conductas, relaciones e interacciones en su interior, determinadas por los miembros que la integran. A través del conjunto de creencias y valores compartidos por los trabajadores de la empresa, se halla la cultura en un alto nivel de abstracción, la cual permite condicionar el comportamiento de la organización, estableciendo su modo de pensar, sentir y actuar. En algunos casos, la rotación escapa del control de la organización cuando el volumen de retiros por decisión de los empleados aumenta notablemente. Cuando el mercado laboral es competitivo y hay una intensa oferta, en general aumenta la rotación de personal, produciendo grandes pérdidas de talento humano y recursos económicos valiosos para la organización. Finalmente, la autora recomienda continuar con la medición de la cultura organizacional y la rotación de personal de manera periódica, a fin de mantener un ambiente laboral adecuado, reforzando su desarrollo y mejorando las posibles falencias que pudieran presentarse evitando pérdidas a largo plazo.

Mejía y otros (2013), plantean que el éxito económico y humano -basado en el talento humanosupone una orientación consciente, pragmática y dirigida a los procesos que llevarán a fortalecer las cualidades necesarias que debe desarrollar el trabajador en su puesto de trabajo. Muchos estudios acogen el concepto del capital humano como el conjunto de capacidades, conocimientos y destrezas de los empleados y directivos de la empresa. Se concluye, que son muchos los elementos que podrían nombrarse como factores esenciales para que una empresa alcance un nivel de desarrollo óptimo, pero el más importante es la integración holística de los empleados con los recursos o herramientas de trabajo que necesitan (tecnologías, sistemas de información, etcétera). La calidad del servicio de una empresa radica en la posibilidad se sostener el talento humano en una línea

PEREZ VASQUEZ, M.A. (2019).

Este es un artículo open Access bajo Licencia Creative Commons Atribución-No Comercial-Sin Derivadas 4.0 Internacional. 
temporal prolongada.

\section{El talento humano en el ámbito empresarial}

El talento humano es un componente esencial en las empresas, dicho cuerpo de trabajo esta guiado por reglas y normas que regulan su funcionamiento, su conducta, su actitud y su responsabilidad; así pues, su actuación reflejará la situación social de la empresa.

La competitividad del talento humano debe ser entendida como aquellas capacidades y habilidades distintivas que poseen cada uno de los empleados y trabajadores, para ejecutar su función de trabajo de la mejor manera posible. También, dicho término hace referencia a que el talento humano debe mantenerse en constante formación, logrando mejorar su desempeño a través de sus valiosos aportes a la organización. El principal desafío para el encargado del talento humano es lograr el bienestar de los colaboradores, conduciéndolos a mejorar los niveles de productividad laboral.

Otro aspecto central dentro del ámbito de la competitividad, es el lugar que ocupa un individuo cualquiera en la organización, entendiendo que existen tareas específicas, responsabilidades, y objetivos organizacionales. El comportamiento organizacional y el ambiente de trabajo son aspectos que favorecen o entorpecen la competitividad del talento humano. La competitividad del talento humano, va de la mano con el hecho de asumir con inteligencia los nuevos retos empresariales, tales como: la globalización, el permanente cambio del contexto y la valoración del conocimiento (Chiavenato, 2001).

También, la competitividad laboral debe estar en concordancia con el proceso de cambio organizacional, ello significa, que más allá de la tarea específica que tiene cada individuo en la organización, existe una función estratégica donde se concibe al trabajador como una fuente de ventaja competitiva sostenida para la empresa. El desarrollo de factores competitivos en el área laboral, lleva consigo, la creatividad, la innovación, el trabajo en equipo, la efectividad y sobre todo apoyo a la capacitación del personal en forma permanente.

El liderazgo, es otro aspecto fundamental que incide en el factor competitivo, debido a que contribuye a fomentar relaciones de confianza, y un clima de respeto, trabajo en equipo, reducción de conflictos, mayor productividad, además de una mayor motivación y satisfacción en el trabajo. La palabra liderazgo se utiliza en el sentido fundamental de aludir al proceso o influencia interpersonal, de llevar a un grupo de personas en una determinada dirección, orientada a la consecución de uno o diversos objetivos específicos por medios no coercitivos.

\section{La gestión de competencias en las empresas}

El termino competencias dentro el ámbito de lo empresarial, está referido a aquellos conocimientos, habilidades y destrezas que facilitan la labor de una persona en su puesto de trabajo, como actividad genérica que contribuye en su realización personal (Yusely, 2018). Las competencias también pueden identificarse, como una capacidad real y medible definida por una empresa, acorde con su función productiva y estructura organizacional.

En cuanto a la medición de las competencias laborales, se hace mención particular de la evaluación del desempeño, instrumento que permite medir y cuantificar los niveles de las competencias laborales en cada uno de los puestos de trabajo, es decir, un indicador que proporciona información acera del desempeño laboral en la organización. Según Ruiz, Gago, García, y López (2013), la evaluación del desempeño es un proceso continuo y sistemático que realiza una empresa en función de medir el valor que un trabajador aporta a la misma. Sin embargo, tal medición no puede ser durante su permanencia en la

PEREZ VASQUEZ, M.A. (2019).

Este es un artículo open Access bajo Licencia Creative Commons Atribución-No Comercial-Sin Derivadas 4.0 Internacional. 
empresa, ni medir todos los aspectos laborales a la vez, sino más bien, una evolución parcial, es decir, en un determinado tiempo o el desempeño del trabajador en una determinada etapa.

Teniendo en cuenta lo anterior, la gestión por competencias, se define como aquella gestión que se realiza en procura de identificar los conocimientos, las habilidades y destrezas de los colaboradores en su puesto de trabajo, además de evaluar su desempeño y potencializar los recursos encaminados al logro de los objetivos misionales de la empresa. En la evaluación del desempeño se lograr evidenciar los problemas o tipos de insuficiencia del trabajador o empleado frente a sus compromisos laborales.

Werther y Davis (2008) consideran, que en función de identificar las competencias se debe realizar un análisis cualitativo del trabajo, ya que tal evaluación está referida a los conocimientos, las habilidades y destrezas para desempeñar con precisión una función laboral.

Por su parte, Mondy (2012), establece que la evaluación de desempeño es un sistema formal de revisión y evaluación y permite direccionar el plan estratégico de la organización.

Por otro lado, según Vera (2016),

La instalación de un modelo de Gestión por Competencias, supone entre otras cosas, un cambio cultural en cuanto a cómo la empresa valora el conocimiento (lo capta, selecciona, organiza, distingue y presenta) y le da importancia a aprender de su propia experiencia y a focalizarse en adquirir, almacenar y utilizar el conocimiento para resolver problemas y aumentar la inteligencia y adaptabilidad de la empresa. La Gestión por Competencias es un proceso o conjunto de ellos que permiten que el capital humano de una organización aumente de forma significativa su satisfacción y comprometimiento con la organización, mediante su gestión de forma eficiente, con el objetivo final de generar ventajas verdaderamente competitivas (p. 20).

Con esta conceptualización sobre la gestión por competencias, se observa la importancia de valorar el conocimiento por parte de la empresa; asimismo, esta invita a que el talento humano se comprometa en mayor grado con la organización. El fortalecimiento del recurso humano a través de la gestión de competencias, es favorable para generar mayores ventajas competitivas en las organizaciones.

Ese mismo autor plantea que:

Los modelos de competencias son una herramienta de gran utilidad para la gestión del talento humano en las empresas, permitiendo un mejor manejo de sus diferentes procesos (selección e incorporaciones, análisis y descripción de cargos, desarrollo y planes de sucesión, remuneraciones y beneficios, entrenamiento, capacitación y evaluación de desempeño) y asegurando que éstos sean consistentes. Además, el desarrollo de un modelo permite tener una visión estratégica y de conjunto de la organización y sus operaciones. La implementación de un modelo propio, genera una cultura de trabajo en la que el personal asume responsabilidades de manera creativa gracias al desarrollo de conocimientos y habilidades (p. 25).

Continuando con los postulados de Vera (2016), la gestión por competencias va a contribuir en los procesos de selección, inclusión, formación y análisis de cargos en las empresas; a su vez, va a permitir que la organización tenga claridad acerca de sus fortalezas al interior del ámbito del capital humano, lo que genera una cultura de trabajo proactivo en la creación de valor en la empresa.

Según lo anterior, Mena (2014), expone que:

PEREZ VASQUEZ, M.A. (2019).

Este es un artículo open Access bajo Licencia Creative Commons Atribución-No Comercial-Sin Derivadas 4.0 Internacional. 
Un modelo de Gestión por Competencias debe comprender la heterogeneidad del perfil de desempeño que tienen los colaboradores, a fin de que ellos puedan alcanzar el nivel óptimo. Este modelo deberá de contemplar las etapas de inserción laboral al igual que las fases de adaptación, junto con el desarrollo de habilidades, destrezas y conocimientos necesarios para lograr eficiencia y eficacia (p.14).

El autor enfatiza en que la gestión por competencia propende por la optimización del desempeño de los colaboradores, apoyado en sus habilidades, destrezas y conocimientos, siendo elementos intangibles generadores de eficiencia y eficacia organizacional.

Para Moreno (2003), Citado por Mejía (2012. P. 41), "las competencias se enmarcan dentro del contexto de la organización y pueden incorporarse a sus prácticas, políticas, sistemas y procesos para ayudar a motivar y apoyar al personal para que su desempeño sea de óptima calidad" (p.41). Se busca la calidad laboral a través de la gestión por competencias.

También se admite que la competencia laboral, "Es la capacidad para colaborar y cooperar con otros, participar activamente de una meta en común, comprendiendo las consecuencias de las propias acciones en el éxito de las metas del área" (Álvarez, 2015, p. 78).

\section{Metodología}

Este trabajo fue de tipo descriptivo, donde se indagó acerca de la importancia que tiene la administración del recurso humano y la gestión por competencias en la eficiencia y la eficacia de las empresas. El estudio se sustenta con información de fuentes secundaria (libros, revistas e información on line).

El método de la investigación fue de tipo deductivo, ya que partir de una generalización del tema propuesto, se hace inferencias particulares acerca del mismo, es decir, con base en la teorización sobre el talento humano, se infiere en aquellos aspectos puntuales acerca de la importancia de la gestión humana en el ámbito de la competitividad empresarial.

\section{Discusión}

En razón de darle una validación empírica a lo expuesto teóricamente, es preciso mencionar una investigación exploratoria, realizada por Pardo y Díaz. (2014), Titulada: "Desarrollo del talento humano como factor clave para el desarrollo organizacional, una visión desde los líderes de gestión humana en empresas de Bogotá D.C."; allí se trató de comprender, cómo los procesos de capacitación y de desarrollo de competencias se convierten en factores clave para la transformación organizacional en un grupo empresas de la ciudad de Bogotá, desde la visión de los líderes de Gestión Humana. Para el desarrollo de esta investigación se aplicó una encuesta dirigida a 81 líderes de talento humano en distintas organizaciones de la ciudad encuestados, $52 \%$ (42) corresponden a empresas medianas de la ciudad de Bogotá, en donde, el $32 \%$ (26) eran pequeñas empresas y $16 \%$ (13) grandes de diversos sectores económicos, y que han tenido procesos de cambio organizacional y han implementado programas de desarrollo del talento humano. Del grupo de empresas estudiadas, $85 \%$ (69) corresponden a empresas privadas y $15 \%$ (12) a empresas públicas.

Los hallazgos evidenciaron, que el $55 \%$ de los directivos de estas empresas, manifestaron que se han presentados efectos favorables luego de la aplicación del modelo de competencias, y de que tales resultados contribuyeron en la mejora del rendimiento de los colaboradores. Por otra parte, la encuesta arrojo una tendencia generalizada de la categoría de desarrollo de personal como parte de los procesos de cambio a nivel organizacional, esto obedece a que las empresas

PEREZ VASQUEZ, M.A. (2019).

Este es un artículo open Access bajo Licencia Creative Commons Atribución-No Comercial-Sin Derivadas 4.0 Internacional. 
encuestadas han desarrollado procesos formativos en sus colaboradores. Sin embargo, los resultados obtenidos también indican la necesidad de revisar los esquemas de gestión de conocimiento que diseñan las empresas analizadas, pues aunque existe un impacto positivo en el desempeño de los colaboradores, se está desaprovechando el potencial de los mismos, al dejar de lado la sensibilización, socialización y transmisión de conocimientos de los procesos para desarrollar competencias previas al cambio, así como la medición permanente, en cuanto a la retroalimentación de los procesos de capacitación orientados al cambio organizacional.

Los resultados obtenidos en dicho estudio, manifiestan la importancia que tiene para cualquier empresa la formación de sus colaboradores. Asimismo, se puede inferir que la gestión por competencias implica procesos de cambio a nivel organizacional, y por tanto, las etapas de formación, cualificación y evaluación de desempeño, son esenciales dentro de los propósitos de dicho modelo, así pues, estos deben estar alineados hacia la competitividad del talento humano en el ámbito empresarial.

\section{Conclusiones}

En conclusión, las competencias laborales no pueden ser analizadas solo dentro del contexto de la individualidad de cada trabajador o empleado. Se deben tener en cuenta varios elementos o componentes concomitantes con la competitividad del talento humano (ambiente laboral, conocimiento, motivación, liderazgo, entre otras). Es claro que el talento humano es un componente clave dentro del contexto empresaria u organizacional, asimismo, debe ser considerado como un factor estratégico, no sólo por el papel que desempeñan en la puesta en práctica de la estrategia empresarial, sino también, por su potencial para convertirse en fuente de ventaja competitiva sostenible.

La administración del recurso humano es un componente clave dentro del contexto empresarial, su importancia se deriva del hecho de relacionar la gestión de los recursos humanos con la estrategia de la organización, significa que tal área ha de ser considerada como un factor estratégico, no sólo por el papel que desempeñan en la puesta en práctica de la estrategia empresarial, sino también por su potencial para convertirse en fuente de ventaja competitiva sostenible.

Se pone en evidencia, que la adecuada aplicación de las prácticas administrativas sobre el componente de recurso humano, dan como resultado una mejor perspectiva de crecimiento empresarial, es decir, a mayor grado de cualificación del recurso humano, mayores serán los beneficios que obtenga una organización en su gestión estratégica empresarial.

Sin duda, uno de los retos es la búsqueda de mayor adaptación del recurso humano a las nuevas formas tecnológicas del mundo, para lograr que sean más eficientes.

Por otra parte, se requiere que aquellos que dirigen las empresas, puedan confiar más en sus colaboradores, que les brinden todos aquellos elementos físicos y emocionales para potencializar el recurso humano, lo que permite que la organización esté preparada para los retos de la competitividad actual.

Se debe motivar, preparar y darle mayor participación al recurso humano en las decisiones estratégicas de la empresa. La creación de los equipos de trabajo siguen siendo claves para el logro del éxito organizacional, no solo por la coordinación de propósitos, sino también, porque se genera un ambiente laboral positivo, que se refleja en una mayor seguridad del recurso humano para ser más competitivos en la era global.

Finalmente, la gestión por competencias permite aumentar la competitividad empresarial, ya que potencializa su mejor recurso, como lo es el talento

PEREZ VASQUEZ, M.A. (2019).

Este es un artículo open Access bajo Licencia Creative Commons Atribución-No Comercial-Sin Derivadas 4.0 Internacional. 
humano, validando sus habilidades, experticias y conocimientos que se reflejara en una mayor productividad laboral.

Se concluye, que el capital humano con una guía adecuada en las organizaciones, puede influir significativamente en el éxito de las mismas y su posicionamiento competitivo en el mercado.

\section{BIBLIOGRAFÍA}

Álvarez, P. (2015). Propuesta para Determinar los Perfiles de Puestos por Competencias en la Gerencia de infraestructura orientado a mejorar la Gestión de Recursos Humanos de la Municipalidad Distrital de Namora - Tesis Pre Grado. Cajamarca, Perú: Universidad Nacional de Cajamarca.

Arevalo Arevalo, A. (2018). Gestión por competencias y gestión administrativa en el Gobierno Regional del Callao. Universidad Cesar Vallejo. Perú.

Bayona C., Goñi, S. y Madorrán C. (2016) Compromiso Organizacional: Implicaciones para la gestión estratégica de los Recursos Humanos. Revista Europea de Dirección y Economía de la Empresa.

Becerra Rodríguez, F. y Álvarez Giraldo C. (2010). El Talento Humano y la Innovación Empresarial en el contexto de las redes empresariales: El Clúster de prendas de vestir en Caldas, Colombia. Universidad Nacional de Colombia sede Manizales.

Criollo Fernández, L. (2014). Clima laboral y rotación de personal motorizado en la Empresa Sodetur S.A. Universidad Central del Ecuador Facultad de Ciencias Psicológicas Carrera de Psicología Industrial

Caicedo L. P., Ausique J. R., Herrera H. y Quenguan S. N. (2013). Universidad del Rosario Facultad de Administración Programa: Administración de Negocios Internacionales. Pamplona, España

Chicaiza Simbaña Y. (2013). La Cultura Organizacional y su incidencia en la Rotación de Personal de Kmeyo.com 2012. Universidad Central del Ecuador. Facultad de Ciencias Psicológicas, Carrera de Psicología Industrial.

Escalona Urquiza, Pablo C. (2015).Administración De Recursos Humanos En Empresa Embotelladora Universidad Nacional Autónoma De México. Facultad De Estudios Superiores Cuautitlán.

García Zambrano, Lidia; García Merino, José Domingo; Rodríguez Castellanos, Arturo (2012). Impacto de la inversión en capital humano sobre el valor empresarial. Academia. Revista Latinoamericana de Administración, núm. 51, pp. 15-26 Consejo Latinoamericano de Escuelas de Administración Bogotá, Organismo Internacional.

Mares Narváez, D. J. y Mendoza Gómez, J. (2015). Causas de rotación de personal en una Empresa de comida rápida en la Región Norte de México. Universidad

Autónoma de Nuevo León. Facultad de Contaduría Pública y Administración, UANL SSN: 2448-5101 Año 2 Número 1 México.

Mejía Giraldo, A., Bravo Castillo, M., y Montoya Serrano, A. (2013). El factor del talento humano en las organizaciones. Ingeniería Industrial/ISSN 1815-5936/Vol. XXXIV/No. 1.

Mejía, Y. M. (2012). Evaluación del desempeño con enfoque en las competencias laborales" para optar el título de Licenciada Psicología Industrial /Organizacional. Guatemala: Universidad Rafael Landívar.

Medina Lorza, A., Delgado Ortega, A., y Lavado Alvarado P. (2012). Fundamentos de un sistema de gestión humana por competencias para soportar la estrategia organizacional en una pyme del sector de la industria de las artes gráficas en Cali (Colombia). Estudios gerenciales, Vol. 28, N0. 122, pp. 121-138.

Miles, R. E. y Snow, C. C. (1978): Organizational strategy, structure, and process, McGraw-Hill Book Cornpany.

Mejía, N. y Puche, L. (2008). Generación de Conocimientos en TIC: Caso UNIGUAJIRA. Recuperado de: Germán A.; Págs. 84,85. http://www.virtualeduca.info/ponencias2013/113/Gene racindeConocimientosenTIC.

PEREZ VASQUEZ, M.A. (2019).

Este es un artículo open Access bajo Licencia Creative Commons Atribución-No Comercial-Sin Derivadas 4.0 Internacional. 
Castillo Dávila N. (2014). Clima, Motivación Intrínseca y Satisfacción Laboral en Trabajadores de Diferentes Niveles Jerárquicos. Pontificia Universidad.

Chiavenato, Idalberto (2001). Administración de Recursos Humanos. Ed. McGraw-Hill; Trad. Villamizar, Germán A.; Págs. 84,85 .

Mejía Giraldo, Armando; Bravo castillo, Mario; Montoya-Serrano Arturo (2013). El factor del talento humano en las organizaciones. Ingeniería Industrial/ISSN 1815-5936/Vol. XXXIV/No. 1

Mena, W. (2014). Propuesta de un Modelo por Competencias orientado al personal administrativo de la Universidad Politécnica Saleciana, sede Cuenca para el año 2014 - Trabajo Post Grado. Cuenca, Ecuador: Universidad Politécnica Saleciana.

Mondy, W. (2012). Capital humano. México: Pearson Educación de México, S.A. de C.V.

Pardo Enciso, C., y Díaz Villamizar, B. (2014) Desarrollo del talento humano como factor clave para el desarrollo organizacional, una visión desde los líderes de gestión humana en empresas de Bogotá D.C. Rev.Suma Neg;5 (11): 39-48. Fundación Universitaria Konrad Lorenz. Elsevier España, S.L.U.

Rivero, A., y Dabos, G. (2017). Gestión diferencial de recursos humanos: una revisión e integración de la literatura. Rev. Estudios Gerenciales 33, 39-51. Universidad ICESI.

Ruiz, E. Gago, M. García, C y López, S. (2013). Recursos humanos y responsabilidad social corporativa. Ciclo formativo grado superior. España: McGraw-Hill/Interamericana Editorial.

Sierra, C. M. (2011). Gestión de Recursos Humanos y retención del capital humano estratégico: Análisis de su impacto en los resultados de Empresas Innovadoras Españolas. España.

Sum Mazariegos, M. I. (2015). Motivación y Desempeño Laboral: Estudio realizado con el personal administrativo de una empresa de alimentos de la zona 1 de Quetzaltenango.

Vera, E. (2015 - 2016). La gestión por competencias y su incidencia en el desempeño laboral del talento humano del banco Guayaquil Agencia Portoviejo. Universidad Técnica.

Werther, W y Davis, K. (2008). Administración de recursos humanos. ( $6^{\text {a }}$ ed.). México, DF: Mc Graw Hill Interamericana S. A. De C.

Yusely Púm Miranda, K (2018). Competencias Laborales y Evaluación del Desempeño. Universidad Rafael Landívar. Guatemala.

PEREZ VASQUEZ, M.A. (2019).

Este es un artículo open Access bajo Licencia Creative Commons Atribución-No Comercial-Sin Derivadas 4.0 Internacional. 